\title{
Reflection-coefficient experimental extraction from S21- parameter for radar oil-spill detection application
}

\author{
Bilal Hammoud ${ }^{1,2 *}$, Fabien Ndagijimana ${ }^{2}$, Ghaleb Faour ${ }^{3}$, Hussam Ayad ${ }^{1}$, Majida Fadlallah', Jalal Jomaah \\ ${ }^{1}$ Lebanese University (LU), Doctoral School of Sciences and Technologies, 1003 Beirut, Lebanon \\ ${ }^{2}$ Grenoble Alpes University (UGA), Grenoble Electrical Engineering Laboratory, 38031 Grenoble, \\ ${ }^{3}$ National Council of Scientific Research (CNRS-L), Remote Sensing Research Center, 22411 Mansouriyeh, Lebanon \\ * Correspondence: bilal.hammoud@minatec.grenoble-inp.fr
}

\begin{abstract}
Oil spill in sea water is one of the main accidents that affect significantly the maritime environment over a long period of time. Knowing the severe influence of oil spills on the ecosystem, it is crucial to have oil spill detecting and monitoring systems for quick intervention and danger containment. In our project, we propose the usage of drones as an oil spill detection system. The drones will be implementing different previously developed multi-frequency approaches for the detection. The effectiveness of such techniques is based on the accuracy of the data collected and their match to the theory. This journal presents a method for the remote extraction of reflection coefficients from multilayer structure modeling an oil spill in sea water. The experimental results for the reflectivity extraction validate the theoretical calculations and allow the implementation of different algorithms based on the statistical information taken directly from the site.
\end{abstract}

Keywords: Oil spill; radar; reflection coefficient; reflectivity; dielectric constant; parameter extraction.

\section{Introduction}

Oil products have become essential for most industrial and commercial mass production. The global need to have petroleum extracts all over the world stresses the in-between continental movements of these products and therefore increases petroleum marine transportation. Sea water oil spills are one of the main accidents effecting the maritime environment over a long period of time. The European Space Agency (ESA) has estimated the annual worldwide spill at more than 4.5 million tons of oil, where $45 \%$ of the amount is due to ship operative discharges ${ }^{[1]}$. Considering that these accidents are happening on a worldwide scale, either intentionally or not, and analysing their severe influence on the ecosystem, it is crucial to have oil spill detecting and monitoring systems for a quick intervention and danger containment. Oil spill detection and monitoring is currently done with the aid of several techniques and sensors, with the most recent ones done remotely using satellite ${ }^{[2-7]}$ and airborne systems ${ }^{[8,9]}$. In 2016, the European Maritime Safety Agency (EMSA) launched the need to complement the satellite maritime surveillance systems -that can detect only $25 \%$ of pollution accidents- by drones ${ }^{[10]}$. In our project, we study the use of drones as an oil spill detection system that will allow a quick assessment of the area where the flag of possible spills is raised by witnesses. Given their relative low costs, multiple drones can be used to scan oil spills in large area during a critical time. Previously, we developed new

Copyright (C) 2018 Bilal Hammoud et al.

doi: $10.18063 /$ som.v3i2.647

This is an open-access article distributed under the terms of the Creative Commons Attribution Unported License

(http://creativecommons.org/licenses/by-nc/4.0/), which permits unrestricted use, distribution, and reproduction in any medium, provided the original work is properly cited. 
dual and multi-frequency advanced algorithms that target oil spill detection for unknown oil thicknesses based on the statistical distribution of the reflectivity values ${ }^{[11,12]}$. The effectiveness of the approach was tested numerically using Monte-Carlo simulations, but to be able to move forward to prototypes and real applications, the results needed to be validated experimentally. Hence, in this journal we present a fast and efficient method for the remote extraction of the power reflection coefficients on multilayer structure modelling an oil spill in sea water.

The rest of this journal is organized as follows: Section 2 presents the oil slick theoretical model. The reflectivity parameter extraction model is described in Section 3. The different parameters used in the theoretical simulation are listed in Section 4-A. The analysis of the theoretical results is presented in Section 4-B. Experimental setup used to obtain the reflectivity measurements in different scenarios is shown in Section 4-C. Experimental results obtained compared to theoretical calculations are analysed in Section 4-D. Finally, Section 5 concludes the journal and stipulates future work.

\section{Theoretical Model}

From the physical point of view, the problem is considered to be a multi-layer structure where we study the reflection of the EM waves from the sea layer covered by a layer of oil. The electrical properties and the physical characteristics are defined for the layers at the boundaries where interaction with EM waves occurs.

We assume that there is an oil slick, with thickness d, on the top of the sea water. An oil spill (with thickness in mm) on the sea surface will dampen the waves and hence reduce the surface roughness ${ }^{[13,14]}$. Furthermore, at open ocean space, with low wind speeds $(2-6 \mathrm{~m} / \mathrm{s})$ (which are considered optimal for oil spill detection $\left.{ }^{[15]}\right)$, the correlation length of the ocean waves is large and the rms-height of the capillary waves is small. Hence, all interfaces are assumed to be very smooth. The sea water is assumed to be very deep so that we neglect the reflections from this medium. The relative dielectric constants (permittivities) of the air, oil and sea water are respectively $\varepsilon_{1}, \varepsilon_{2}$ and $\varepsilon_{3}$. These permittivities could be complex or real. The different mediums are assumed to be non-magnetic, so that the permeability $\mu=1$, which allows us to write the refractive indices " $\mathrm{n}$ " for the different materials as $n_{\mathrm{i}}=\sqrt{\varepsilon_{\mathrm{i}}}$. The electromagnetic (EM) signals are normally incident on the oil surface interface. The field reflection coefficient from the interface between two mediums $\mathrm{p}$ and $\mathrm{q}$ is given by

$\rho_{\mathrm{pq}}=\frac{\mathrm{n}_{\mathrm{p}}-\mathrm{n}_{\mathrm{q}}}{\mathrm{n}_{\mathrm{p}}+\mathrm{n}_{\mathrm{q}}}$

The formula of the reflectivity (power reflection coefficient) for the three-layer structure is previously developed ${ }^{[11]}$. It is given by

$\mathrm{R}=|\rho|^{2}=\frac{\rho_{12}^{2}+\rho_{23}^{2}+2 \rho_{12} \rho_{23} \cos (2 \delta)}{1+\rho_{12}^{2} \rho_{23}^{2}+2 \rho_{12} \rho_{23} \cos (2 \delta)}$

$\delta$ is the phase shift that is dependent on the oil-refractive index $\mathrm{n} 2$, the frequency of the electromagnetic wave (reflected in its wavelength defined as $\lambda_{0}=\mathrm{c} / f$ where $\mathrm{c}$ is the speed of light) and the thickness of the oil layer d. It is given by

$\delta=\frac{2 \pi}{\lambda_{0}} n_{2} d$

The reflectivity $\mathrm{R}$ is a trigonometric function with period TR that is dependent on the oil-refractive index and the frequency of the electromagnetic wave. The period is expressed as

$T_{R}=\frac{2 \pi}{\frac{2 \delta}{d}}=\frac{\lambda_{0}}{2 \sqrt{\varepsilon_{2}}}$

The reflectivity in oil-free scenario is calculated using

$R_{\text {water }}=\left(\frac{n_{1}-n_{3}}{n_{1}+n_{3}}\right)^{2}$

\section{Parameter Extraction Model}




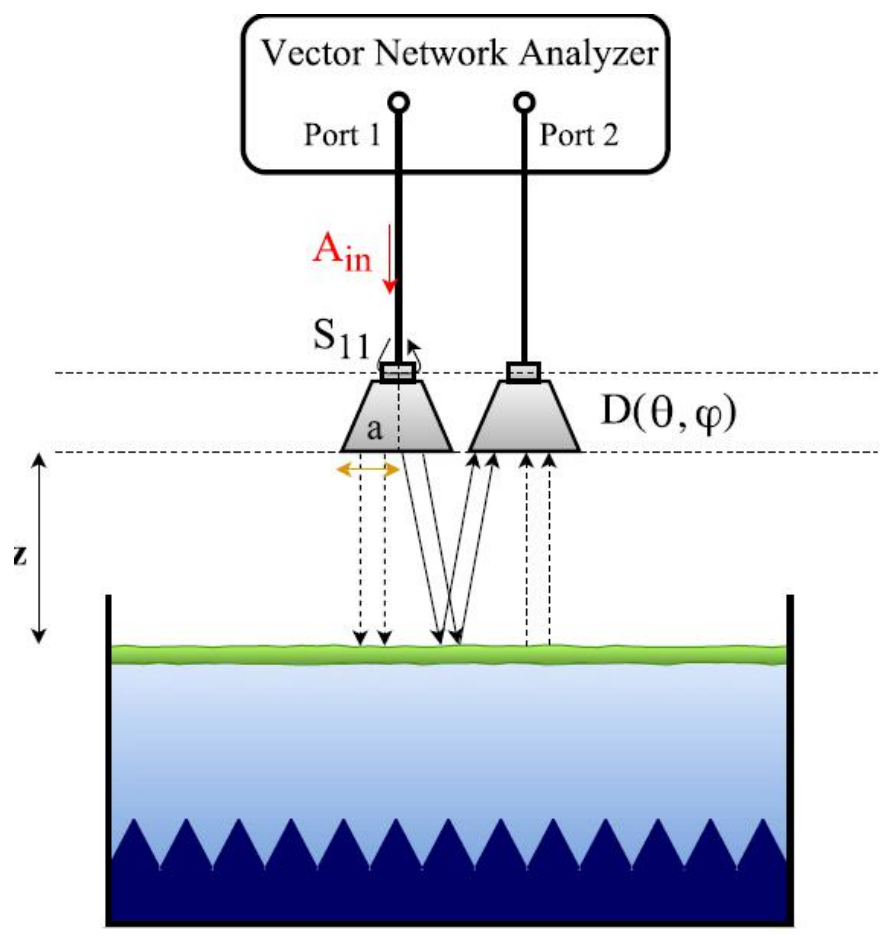

Figure 1. Experimental setup for reflection coefficient parameter extraction.

Figure 1 shows the experimental setup used to extract $\rho$, the multilayer reflection coefficient parameter. Two antennas are connected to a Vector Network Analyser (VNA). Ports 1 and 2 are assumed to be the transmission (input) and reception (output) ports for the electromagnetic waves. $A_{\text {in }}$ represents the amplitude of the input EM signal to the system. $\mathrm{D}_{\mathrm{u}}(\theta, \varphi)$ represents the directivity of the antenna $\mathrm{u}$, where $\theta$ and $\varphi$ represent the radiative pattern angles in elevation and azimuth respectively. $S_{11}$ and $S_{22}$ are the antennas return losses, i.e, how much of power is reflected from the antenna itself to the port where it is connected. $z$ represents the distance between the antennas and the surface to be characterized. The system in front of the antennas models a multilayer structure composed of air, oil and water. It is composed of a basin filled with water, and covered by a layer of oil. Since in our model we assume a deep ocean, the deep distance between the surface and the ground allows us to neglect any effect of the reflected EM waves from the last medium. For this reason, we fix microwave absorbers on the base of the basin to absorb the transmitted electromagnetic waves into the water. $S_{21}$ representing the transmission parameter of the EM waves from antenna 1 to antenna 2 can be expressed as

$\mathrm{S}_{21}=\mathrm{A}_{\mathrm{in}} \cdot\left(1+\mathrm{S}_{11}\right) \cdot \mathrm{D}_{1}(\theta, \varphi) \cdot \mathrm{e}^{-\mathrm{jk} \mathrm{l}_{1}} \cdot \rho \cdot \mathrm{e}^{-\mathrm{jk} \mathrm{l}_{2}} \cdot \mathrm{D}_{2}(\theta, \varphi) \cdot\left(1+\mathrm{S}_{22}\right)$

with $\mathrm{I}$ being the path distance traversed by the EM from the antenna to the medium. If we place a plate of perfect conductor metal at the same distance $\mathrm{z}$ far away from the antennas, then we obtain

$\mathrm{S}_{21, \mathrm{~m}}=\mathrm{A}_{\mathrm{in}} \cdot\left(1+\mathrm{S}_{11}\right) \cdot \mathrm{D}_{1}(\theta, \varphi) \cdot \mathrm{e}^{-\mathrm{jk} \mathrm{l}_{1}} \cdot \rho_{\mathrm{m}} \cdot \mathrm{e}^{-\mathrm{jk} \mathrm{l}_{2}} \cdot \mathrm{D}_{2}(\theta, \varphi) \cdot\left(1+\mathrm{S}_{22}\right)$

where $\left|\rho_{m}\right|=1$ for perfect conductor metal interface. This means that the metal acts as a total reflector and the metal placement is equivalent to system calibration. In other words, $S_{21, m}$ is allowing us to ignore the need to exactly identify the value of each parameter of (6), because using it as a normalization factor is actually providing the value of the reflection coefficient $\rho$ as

$\frac{S_{21}}{S_{21, m}}=\frac{\rho}{\rho_{\mathrm{m}}}=|\rho| \angle \rho+180^{\circ}$

Using this model, we can investigate the effect of many parameters on the performance of the overall system, including:

1) the minimum required power for this kind of applications by studying the effect of the power sent (using Ain).

2) The influence of the antennas radiation patterns and its directivity on the study analysis. This effect can be studied by varying the distance $\mathrm{z}$ between the antennas and the oil/water surface and by varying the angle of incidence of the EM waves.

3) The effect of the several paths of transmission and reflection.

4) The effectiveness of the absorbers used at the bottom: how much these absorbers are preventing reflected EM 
waves from the last medium as to have the model as realistic as possible to that of a deep ocean.

5) The influence of the surface roughness on the accuracy of the parameter extraction.

\section{Results}

\section{A. Simulation Setup}

As shown in (2) and (4), the reflectivity function is periodic where its period is dependent on the frequency of the electromagnetic wave used and the oil dielectric constant. With respect to the target application related to oil spill detection, oil property is not a design-controlled parameter. Therefore, in the following, the study will be based for realistic range of oil dielectric constant and for all the range of possible oil thickness. What is a design-controlled parameter is actually the number of frequencies chosen during analysis and algorithm development. That said, the analysis of the reflectivity will be studied with respect to the variation of the frequencies and the oil thickness to eventually reach the best decision as to which frequencies should be used for the oil detection.

Our model is a planar multilayer structure composed of three consecutive mediums: air, oil and water. For the air, it is assumed that $\varepsilon_{1}=1 \mathrm{~F} / \mathrm{m}$. For the oil, depending on its type, the real part of its dielectric constant varies between 2.9 and $3.3 \mathrm{~F} / \mathrm{m}$. However, the imaginary part is very small (of order $0.01 \mathrm{j}$ ) and can be neglected without any influence on the results. As for the sea water, its dielectric constant, $\varepsilon_{3}$, is function of the water temperature $t_{w}$, water salinity $s_{w}$ and the frequency of the electromagnetic signal used. For its calculation, we use the model with $t_{w}=20^{\circ} \mathrm{C}$ and $\mathrm{s}_{\mathrm{w}}=35$ $\mathrm{ppt}^{[16]}$. The oil slick is considered to be thick and dispersed on top of the water over all the area of study. The thickness of the oil slick is varied between 0 and $10 \mathrm{~mm}$. The electromagnetic waves are transmitted normally to the surfaces. The frequencies used for the EM vary between $4 \mathrm{GHz}$ and $12 \mathrm{GHz}$, but only specific frequencies will be displayed in the results.

\section{B. Simulation Results Analysis}

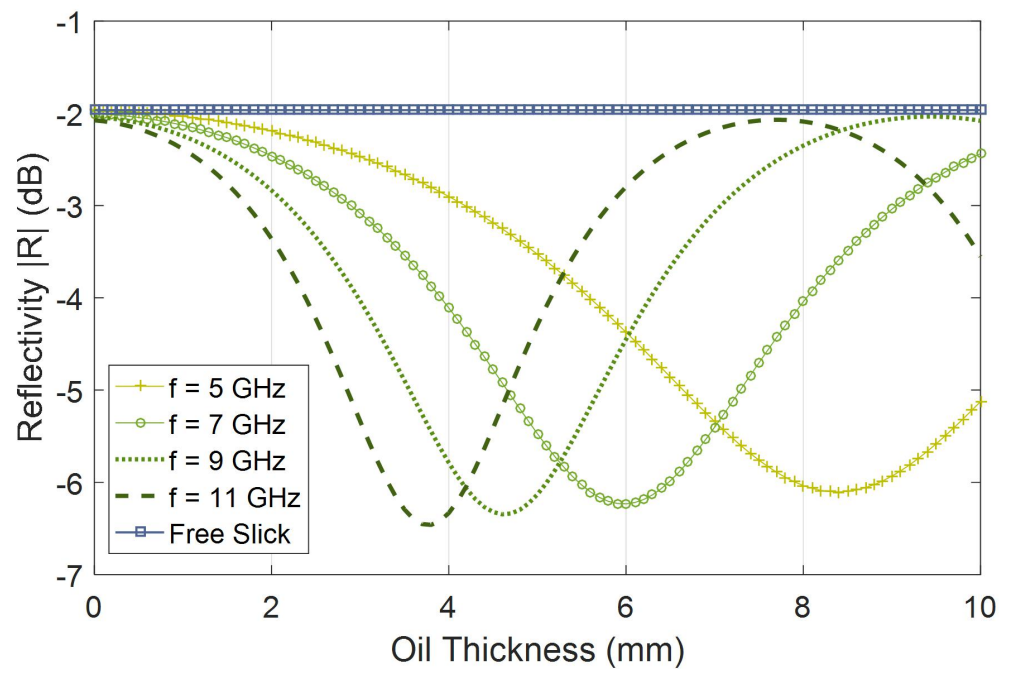

Figure 2. Reflectivity $\mathrm{R}$ (in $\mathrm{dB}$ ) versus oil thickness (in $\mathrm{mm}$ ) at different frequencies with " $2=3 \mathrm{~F} / \mathrm{m}$.

Figure 2 shows the reflectivity values for different frequencies $(5,7,9$ and $11 \mathrm{GHz})$ versus the oil thickness variation. For $\mathrm{d}=0 \mathrm{~mm}$, no oil exists and it is pure water. In this case, the reflectivity values are independent of the oil thickness and constant for every frequency. This behaviour is shown by the horizontal blue plot. This plot is helpful for the comparison of results and will be a reference plot that is used to study the reflectivity behaviour in the presence of an oil slick. The deviation from this plot will give the variation in power ratio, which in turn will show how accurate our system is in terms of oil spill detection.

The reflectivity is a trigonometric function. Increasing the frequency will decrease its period. This behaviour is observed when comparing the four plots of reflectivity at different frequencies, where $\mathrm{f}=11 \mathrm{GHz}$ and $\mathrm{f}=5 \mathrm{GHz}$ record periods of $7.8 \mathrm{~mm}$ and $17.3 \mathrm{~mm}$ respectively. Between 0 and $8 \mathrm{~mm}$, the reflectivity values at $5 \mathrm{GHz}$ are decreasing in a 
monotonic behaviour with a very small slope at $(0$ and 2) $\mathrm{mm}$. This slope increases for the rest of the range ( 2 and 7$)$ $\mathrm{mm}$ and then decreases again until it reflects at $8.6 \mathrm{~mm}$. The main drawback for this plot is that for small thickness layers of oil, any error in the power reflectivity estimation would disrupt oil detection due to the very small variation of the power difference between the oil free scenario and the oil presence scenario. In contrast, the variation in the reflectivity values at $(7,9$ and 11$) \mathrm{GHz}$ is quite high for consecutive values of oil thickness, and it allows oil detection at small thickness values. However, the periodicity of such signals would again disrupt detection for higher thickness levels (for example at $\mathrm{d}=7.8 \mathrm{~mm}$ at $11 \mathrm{GHz}$ ). Here comes the idea of using algorithms where a combination of frequencies for the oil detection is implemented as developed ${ }^{[11,12]}$.

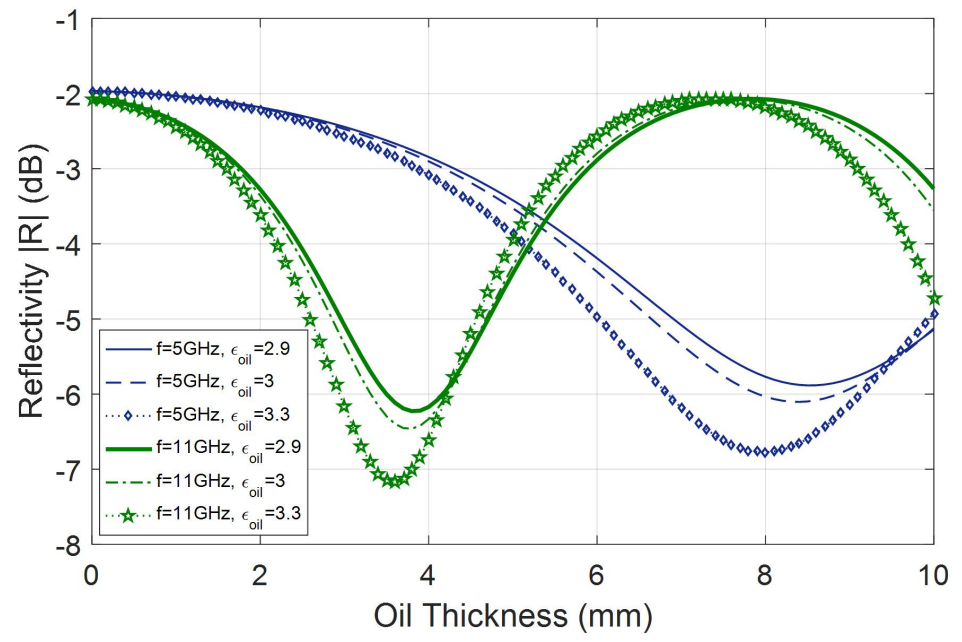

Figure 3. Reflectivity R (in $\mathrm{dB}$ ) versus oil thickness (in $\mathrm{mm}$ ) at different frequencies and different oil dielectric constants

Figure 3 presents the reflectivity values for different oil dielectric constants $\left(\varepsilon_{2}=2.9,3\right.$ and 3.3$) \mathrm{F} / \mathrm{m}$ at two frequencies (5 and 11) GHz. For both frequencies, the difference in the dielectric constant values does not modify the reflectivity values at small thicknesses $(0-2) \mathrm{mm}$. However, for higher thickness values, the reflectivities for both frequencies start to change for different dielectric constants. At $5 \mathrm{GHz}$, the difference between the reflectivities when $\varepsilon_{2}=2.9 \mathrm{~F} / \mathrm{m}$ and $\varepsilon_{2}=3.3 \mathrm{~F} / \mathrm{m}$ reaches approximately $1 \mathrm{~dB}$ at around $7.8 \mathrm{~mm}$. At $11 \mathrm{GHz}$, the difference is high at around $3.5 \mathrm{~mm}$, decreases to null at $7.8 \mathrm{~mm}$ and increases again to $1.5 \mathrm{~dB}$ at $10 \mathrm{~mm}$. From the results discussed above, we notice that although at different thickness values there exists some noticeable difference in reflectivity values at the same frequency for different dielectric constants. Yet, this difference occurs far below the water reflectivity value $(-2$ $\mathrm{dB})$. Therefore, the variation in the oil dielectric constants should not lead to oil detection algorithm failure. Rather, it leads to an increase in the error of correctness percentage, which is still acceptable. In other words, although we may not know the exact value of the oil dielectric constant, we should still be able to use the different algorithms to have a correct decision.

\section{Experimental Setup}

For the experimental setup, we used a basin with dimensions of $38.5 \times 26.5 \times 22.5$ in length, width and height respectively, all in $\mathrm{cm}$. At the bottom of this basin, we put $5 \mathrm{~cm}$ of absorbers. Having efficient absorption from the bottom level, it was enough to fill the basin with only $6 \mathrm{~cm}$ of water. On the top of the water surface, we spilled olive oil which has a dielectric constant very similar to that of crude fuel oils shipped between countries ${ }^{[17]-[19]}$. The volume of oil spilled corresponds to a thickness of $3 \mathrm{~mm}$ dispersed over all the area in the basin. For the EM waves transmission and reception, we used two horn antennas. The dimension "a" of the antenna is around $7 \mathrm{~cm}$. The two antennas were displaced $4.5 \mathrm{~cm}$ away from each other, and the inclination above a horizontal plan for each antenna is around $2 \mathrm{~cm}$. The inclination and the antennas dimensions make around $7.5^{\circ}$ antenna tilt with vertical plane. The antennas are placed $10 \mathrm{~cm}$ above the basin. This distance is considered good enough for the range of frequencies used in study.

System calibration is done using a perfect metal plate placed at the same height as that of the oil surface. Taking 
into account the antennas dimensions, the distance between these antennas and the oil surface should be large enough to ensure that we are working in normal transmission and reception. In addition, this distance is critical to decouple the antennas and the medium of EM waves-surfaces interactions. However, since we are doing calibration using perfect metal material at the same height and the same antennas setup, and since our analysis are based on the average power poynting vector measured by the VNA, the effect of the small distance used in experiment will not touch the parameter extraction precision. Furthermore, the angle of incidence $7.5^{\circ}$ is used for this distance to make sure that the reflections from the surface will fall directly in the second antenna. This scenario very much resembles very much the scenario where the antennas will be placed at a higher distance with a smaller angle of incidence (around $2^{\circ}$ ). Note that the small angle of incidence in the experiment can be neglected in terms of its effect on the reflectivity, and the experimental results will be compared to the simulation results with perfect normal incidence.

\section{Experimental Results Analysis}

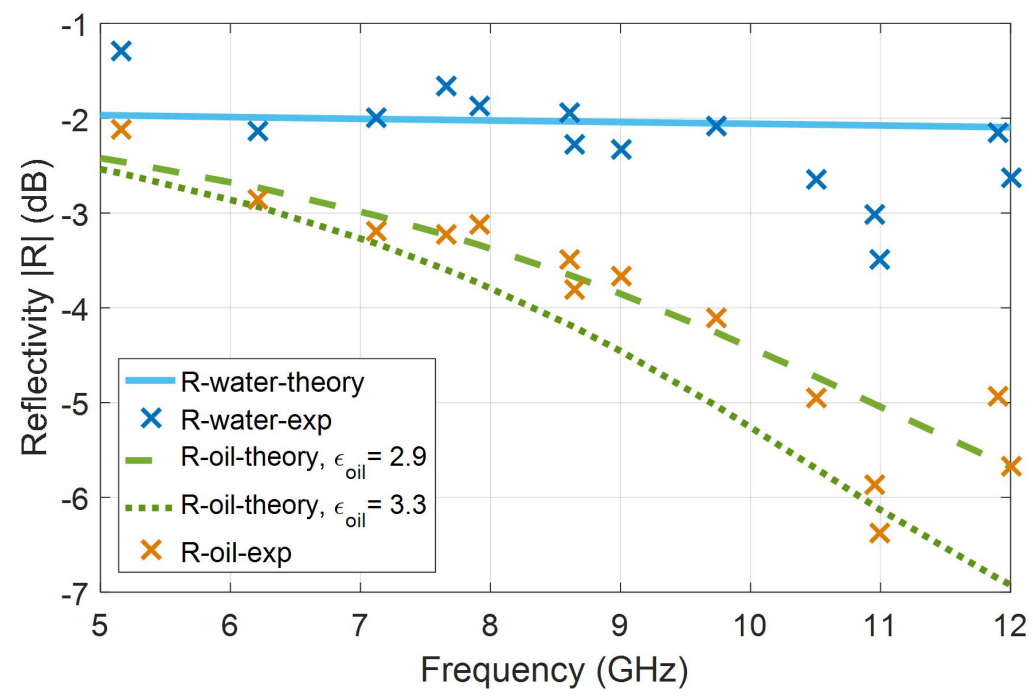

Figure 4. Comparison between theoretical and experimental reflectivity values at different frequencies with oil thickness $\mathrm{d}=3 \mathrm{~mm}$

Figure 4 shows the comparison between theoretical and experimental reflectivity values at different frequencies for both scenarios, oil-free slick and oil-slick with thickness $d=3 \mathrm{~mm}$. For the oil-free slick, experimental values approximately fit the theoretical values with a small error (less than $0.2 \mathrm{~dB})$ in the $(6-10) \mathrm{GHz}$ range of frequency. This error is bigger at 5, 10.5 and $12 \mathrm{GHz}$ and it is maximal at $11 \mathrm{GHz}$ where it reaches around $1.2 \mathrm{~dB}$. The experimental water reflectivity values confirm the hypothesis with the frequency change; all values obtained are varying in a horizontal trend. With respect to oil presence scenario, the experiment confirms the decrease of the reflectivity values from $-2 \mathrm{~dB}$ to reach a minimum of $-6.3 \mathrm{~dB}$ at $11 \mathrm{GHz}$. The obtained experimental values are almost within the region countered by the two oil reflectivity plots with dielectric constants 2.9 and 3.3, which is enough for an oil spill detection application, where the dielectric constants of the oil are not exactly unknown. Furthermore, although at high frequencies $(11$ and $12 \mathrm{GHz})$ the experimental values present the highest error $(1 \mathrm{~dB})$ from the theoretical value, this will not move the data to the region where it could be misinterpreted as oil-free region. 


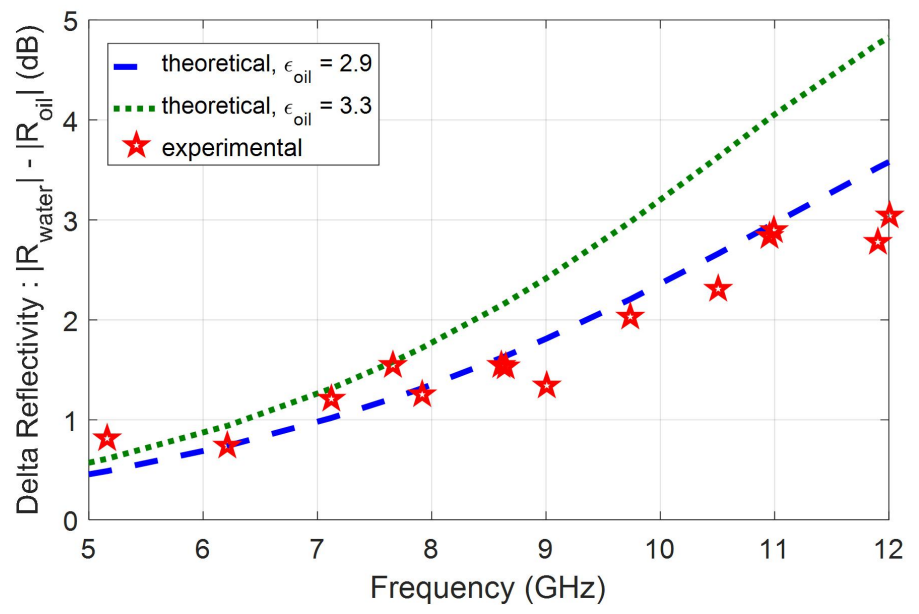

Figure 5. Comparison between theoretical and experimental oil reflectivity values calibrated by experimental water reflectivity

Figure 5 shows the oil reflectivities calibrated by the experimental water reflectivities. This calibration can be done by having measurements of known clean sea water and candidate oil spill regions. Compared to the theoretical values, the results show a maximum error of $0.5 \mathrm{~dB}$. Furthermore, we notice that the experimental values almost fit with the reflectivity values for oil with dielectric constant $\varepsilon_{2}=2.9 \mathrm{~F} / \mathrm{m}$ which can give an indication of the present oil dielectric constant. Experimental results in Fig. 4 and 5 show that based on reflection coefficient parameter extraction, it is possible to perform oil spill detection that can be implemented using different advanced algorithms as mentioned in ${ }^{[1]}$ and $^{12]}$. In addition, this technique may be used to remotely estimate the oil dielectric constant for material characterization, but this is beyond the scope of this journal.

\section{Conclusion}

In this journal, we experimentally demonstrate the use of reflection coefficient parameter extraction for radar application. Oil spill in sea water is modelled as a multilayer structure with planar interfaces. EM waves transmitted to the oil-water model are reflected back and processed for reflection coefficient parameter extraction. For different oil thicknesses, the reflectivity values vary with the EM frequency range, which allows the distinction between 'oil presence' and 'oil absence' on sea water. The results experimentally obtained verify the simulated results. Having the experimental data validated, it is now possible to use it as input data to multi-frequency advanced algorithms that target oil spill detection for unknown oil thickness based on the statistical distribution of the reflectivity values.

\section{Funding and Acknowledgments}

This work was supported by the National Council of Research at Lebanon (CNRS-L) and the Lebanese University.

\section{References}

1. ESA. Oil pollution monitoring. Remote Sensing Exploitation Division. p. 2, ESRIN- European Space Agency.

2. Laneve G, Luciani R. Developing a satellite optical sensor based automatic system for detecting and monitoring oil spills. Environment and Electrical Engineering (EEEIC), 2015 IEEE 15th International Conference on, pp. 1653-1658, IEEE, 2015.

3. Dan W, Jifeng S, Yongzhi Z, et al. Application of the marine oil spill surveillance by satellite remote sensing. Environmental Science and Information Application Technology, 2009. ESIAT 2009. International Conference on, vol. 1, pp. 505-508, IEEE, 2009.

4. Rocca F. Remote sensing from space for oil exploration. Geoscience and Remote Sensing Symposium (IGARSS), 2015 IEEE International, pp. 2876-2879, IEEE, 2015.

5. Minchew B, Jones CE, Holt B. Polarimetric analysis of backscatter from the deepwater horizon oil spill using 1-band synthetic aperture radar. IEEE Transactions on Geoscience and Remote Sensing 2012; 50(10): 3812-3830.

6. Frost JD, Barnes CF. Assessment and enhancement of sar noncoherent change detection of sea-surface oil spills. IEEE Journal of Oceanic Engineering 2018; 43(1): 211-220.

7. Xu L, Wong A, Clausi DA. An enhanced probabilistic posterior sampling approach for synthesizing sar imagery with sea ice and oil spills. IEEE Geoscience and Remote Sensing Letters 2017; 14(2): 188-192. 
8. Collins MJ, Denbina M, Minchew B, et al. On the use of simulated airborne compact polarimetric sar for characterizing oil-water mixing of the deepwater horizon oil spill. IEEE Journal of Selected Topics in Applied Earth Observations and Remote Sensing 2015; 8(3): 1062-1077.

9. Hensley S, Jones C, Lou Y. Prospects for operational use of airborne polarimetric sar for disaster response and management. Geoscience and Remote Sensing Symposium (IGARSS), 2012 IEEE International, pp. 103-106, IEEE, 2012.

10. Lecomte E. En fevrier 2017, des drones vont traquer la pollution maritime, 2017.

11. Hammoud B, Mazeh F, Jomaa K, et al. Multi-frequency approach for oil spill remote sensing detection. High Performance Computing \&amp; Simulation (HPCS), 2017 International Conference on, pp. 295-299, IEEE, 2017.

12. Hammoud B, Mazeh F, Jomaa K, et al. Dual-frequency oil spill detection algorithm. Computing and Electromagnetics International Workshop (CEM) 2017, pp. 27-28, IEEE, 2017.

13. Skrunes S, Brekke C, Eltoft T. Oil spill characterization with multi-polarization c-and x-band sar. Geoscience and Remote Sensing Symposium (IGARSS), 2012 IEEE International, pp. 5117-5120, IEEE, 2012.

14. Marzialetti P, Laneve G. Oil spill monitoring on water surfaces by radar 1, c and $\mathrm{x}$ band sar imagery: A comparison of relevant characteristics. Geoscience and Remote Sensing Symposium (IGARSS), 2016 IEEE International, pp. 7715- 7717, IEEE, 2016.

15. Jha MN, Levy J, Gao Y. Advances in remote sensing for oil spill disaster management: State-of-the-art sensors technology for oil spill surveillance. Sensors 2008; 8(1): 236-255.

16. Ulaby FT, Long DG, Blackwell WJ, et al. Microwave radar and radiometric remote sensing, vol. 4. University of Michigan Press Ann Arbor, 2014.

17. Vrba J, Vrba D. Temperature and frequency dependent empirical models of dielectric properties of sunflower and olive oil. Radioengineering 2013; 22(4): 1281-1287.

18. Maruska HP, Forster EO. Dielectric processes in heavy oils. Electrical Insulation \&amp; Dielectric Phenomena-Annual Report 1984, Conference on, pp. 334-342, IEEE, 1984.

19. Muntini MS, Pramono YH, Minarto E, et al. Modeling and simulation of microwave propagation on crude oil heating. Sensors, Instrumentation, Measurement and Metrology (ISSIMM), 2017 International Seminar on, pp. 46-50, IEEE, 2017. 